\title{
A dosimetric study of electron beam therapy vs. high-dose-rate mould brachytherapy in adjuvant treatment of non-melanoma skin carcinomas of the head and neck region
}

\author{
Souransu Sen, MBBS, Anis Bandyopadhyay, MBBS, MD, DNB, Jayanta Kumar Pal, MSc DRP, Arnab Kumar Ghosh, MBBS, \\ Asit Ranjan Deb, MBBS, DGO, MD, DNB \\ Department of Radiotherapy, Medical College Kolkata, India
}

\begin{abstract}
Purpose: Radiation for superficial tumours of the head and neck region can be given either by brachytherapy or electrons. Brachytherapy (BT), due to rapid dose fall-off and minor set-up errors, should be superior to external beam radiotherapy (EBRT) for treatment of lesions in difficult locations such as the nose and earlobe. The present study is a dosimetric comparison of computed tomography (CT)-based mould brachytherapy treatment plans with 3D conformal electron beam therapy in the treatment of non-melanoma skin cancers (NMSC).

Material and methods: From December 2017 to November 201810 patients with NMSC of the head and neck region (forehead, nose, cheek) who underwent adjuvant radiation with HDR brachytherapy (BT) with a surface mould individual applicator were enrolled for analysis. We evaluated dose coverage by minimal dose to $90 \%$ of planning target volume (PTV, $\left.\mathrm{D}_{90}\right)$, volumes of PTV receiving 90-150\% of prescribed dose (PD) $\left(\mathrm{VPTV}_{90-150}\right)$, conformal index for $90 \%$ and $100 \%$ of PD $\left(\mathrm{COIN}_{90}, \mathrm{COIN}_{100}\right)$, dose homogeneity index (DHI), dose nonuniformity ratio (DNR), and exposure of organs at risk (OARs) (eyes, lens, underlying bone and skin). Prospectively, we created CT-based treatment plans for electron beam therapy. We compared conformity $\left(\mathrm{COIN}_{90}, \mathrm{COIN}_{100}\right)$, dose coverage of PTV $\left(\mathrm{D}_{90}, \mathrm{VPTV}_{90}, \mathrm{VPTV}_{100}\right)$, volumes of body receiving $10-90 \%$ of $\mathrm{PD}\left(\mathrm{V}_{10}-\mathrm{V}_{90}\right)$, doses to OARs $\left(\mathrm{D}_{0.1 \mathrm{cc}}\right.$ and $\left.\mathrm{D}_{2 \mathrm{cc}}\right)$ of $\mathrm{BT}$ and electron plans.

Results: We obtained mean BT-DHI 0.81, BT-DNR 0.608, Electron-DHI 1.25. We observed no significant differences in $\mathrm{VPTV}_{90,100}$ and $\mathrm{D}_{90}$ between BT and electron beam. Mean BT-VPTV 125,150 were significantly higher than Electron-VPTV 100,125 . COIN $_{90}$ was superior for BT plans.

Conclusions: CT-based surface mould brachytherapy results in better conformity of superficial lesions on small, irregular surfaces such as the nose and inner canthus than electrons with a slightly higher skin dose.
\end{abstract}

J Contemp Brachytherapy 2019; 11, 6: 547-553

DOI: https://doi.org/10.5114/jcb.2019.90233

Key words: surface mould brachytherapy, electron beam, head and neck.

\section{Purpose}

Non-melanoma skin cancers (NMSCs) comprise largely squamous cell carcinoma (SCC) and basal cell carcinoma (BCC). The global incidence of NMSCs was estimated to be around 1 million in 2018 [1]. The NMSCs comprise $1-2 \%$ of cutaneous neoplasms in Indians, of which $83.9 \%$ were confirmed as SCC and $16.1 \%$ as BCC $[2,3]$.

The primary treatment of choice for NMSCs is surgery. Radiation therapy (RT) can be used as a primary treatment for patients disqualified from surgery, patients in whom good cosmetic and functional results cannot be achieved by surgery or patients with lesions associated with clinical perineural invasion (PNI) with gross tu- mour extending to sites that render complete resection unlikely or unfeasible, such as the cavernous sinus [4]. Postoperative RT can be added in presence of adverse pathological features such as close or positive margins, PNI, cartilage or bone invasion, as it reduces local recurrences [5].

Radiation therapy in these cases can be delivered by a number of modalities consisting of high dose rate (HDR) brachytherapy (BT) or external beam RT (EBRT). Among EBRT techniques orthovoltage RT with beam energies in the range $100-250 \mathrm{kV}$ or electrons or high energy photons can be used [5]. Among the above modalities, the most commonly used modalities are electrons and surface mould brachytherapy. 
Electron beam therapy often requires templates and lead cutouts, the construction of which can be messy and uncomfortable for the patient when using skin collimation for better dose coverage. In the treatment of small fields, electron dosimetry requires special attention because the percentage depth dose (PDD) and output change significantly according to the specific block shape and size [6]. HDR BT, despite the more heterogeneous dose distribution, presents several advantages, particularly for irregular surfaces and challenging setups [6]. Reports of older published series mainly done in European facilities with wide experience in BT showing efficacy in treating skin cancer have led to recent interest and growth in new techniques $[7,8,9]$. Several innovative applicators have been introduced to the BT community, and the use of skin BT has significantly increased [10].

Surface applicators have been used in radiotherapy since the turn of the $20^{\text {th }}$ century. Applicators such as wax and paraffin skin custom moulds were developed and used with radium needles or radon seeds to cure skin cancer [11]. The advantages of brachytherapy over electrons are a rapid dose fall-off outside the target volume and short duration of treatment $[6,12,13,14,15]$. Moreover, with respect to difficult areas such as in and around the nose or earlobe, major disadvantages of electron or EBRT are the setup errors and difficulty in planning in small, irregular fields and so a significant PTV margin must be given [12].

Although BT should be better logically, dosimetric studies comparing BT and electrons for treatment of superficial skin tumours, especially in difficult areas such as the nose, are lacking. Moreover, dosimetric studies of BT with 3D planning are even more scarce. The purpose of this study was to make a dosimetric comparison of computed-tomography based (CT-based) mould brachytherapy treatment plans with 3D conformal electron beam treatment plans in the treatment of NMSC of the head and neck region, especially in difficult sites over and around the nose.

\section{Material and methods}

\section{Patient selection and patient characteristics}

Patients with NMSCs (BCC or SCC) of the head and neck region, who received adjuvant radiation in the Department of Radiotherapy, Medical College and Hospi-

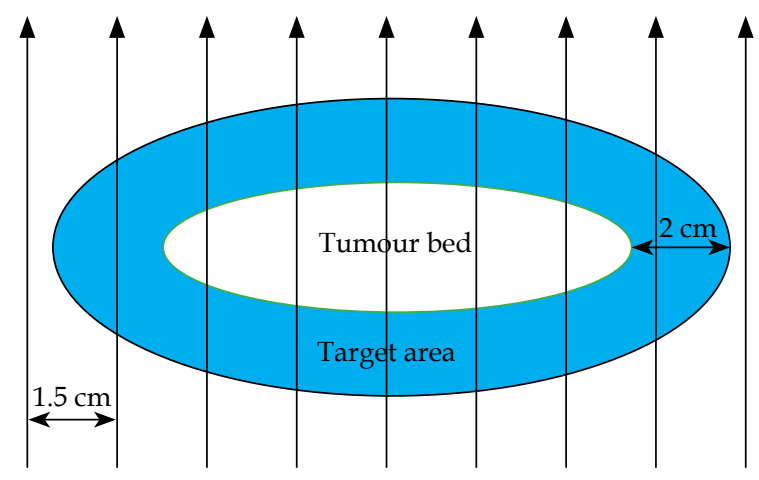

Fig. 1. Schematic diagram showing the relation of tumour bed, target area and the arrangement of plastic catheters tal, Kolkata, between December 2017 and November 2018 with HDR BT using individual surface mould applicators, were enrolled for this study. The patients who were selected had received only HDR BT treatment to the local site as part of their adjuvant treatment and did not require any nodal irradiation.

A total of 10 patients were selected and analysed. The patients were aged between 40 and 75 years (median age of 56.6 years). Of the 10 patients, 6 patients had histologically proven basal cell carcinoma and 4 patients had squamous cell carcinoma. Out of the 10 patients, in 7 patients the lesion was located over the ala of the nose and adjacent cheek, in 2 patients the lesion was over the nose proper and in 1 patient the lesion was over the root of the nose. All of the patients had undergone radical resection followed by adjuvant radiation with HDR brachytherapy using individual surface mould applicators. Among the patients, 9 received adjuvant therapy owing to a close margin and 1 patient needed radiation due to evidence of cartilage invasion. None of the patients had any positive nodes or any evidence of distant metastasis at the time of treatment. None of the patients had indications for regional nodal irradiation.

\section{Details of brachytherapy treatment received by the patients}

The tumour bed was marked over the scar in correlation with preoperative images. An area beyond the bed with a $2 \mathrm{~cm}$ margin all around was marked as the target area (Figure 1). The patient was then immobilised using a three clamp head and neck thermoplastic mask. The target area was redrawn over the mask. On the thermoplastic mask layers of dental wax (of about $5 \mathrm{~mm}$ thickness) were applied to adequately cover the target area. Grooves were created over the wax with $1.5 \mathrm{~cm}$ separation and flexible HDR catheters of adequate length were then fixed to the mould, one on each of these grooves. Additional layers of dental wax were applied to cover the catheters and to give stability to the mould (Figure 2). To ensure proper dose delivery on the borders of the target, the number of catheters was selected to cover the whole target volume with the margin.

Before acquiring the CT scan, the target area was marked with thin copper wire. CT scan (BrillianceCT-16 slice with Accusim Virtual simulation; Philips Health Care Solutions, DA Best, The Netherlands) was performed using a thin slice width of 1-2 $\mathrm{mm}$ from the vertex to just below the chin $(120 \mathrm{kV}$ and $250 \mathrm{mAs})$. After CT simulation the data were transferred to the treatment planning system (TG-43 compliant BrachyVision TPS, Eclipse Version 13.5, Varian Medical Systems, Palo Alto, CA, USA) computer. Then following image reconstruction, the planning target volume (PTV) corresponding to the target area was contoured. The skin and soft tissue underlying the target area formed the depth of the PTV. A small additional margin was taken for the PTV to account for setup errors. The organs at risk (OARs: underlying bones, eyeballs, lens and skin) were also contoured and the dose was prescribed. After that, optimisation was done such that the $100 \%$ isodose line conformed to the PTV as much as possible. 

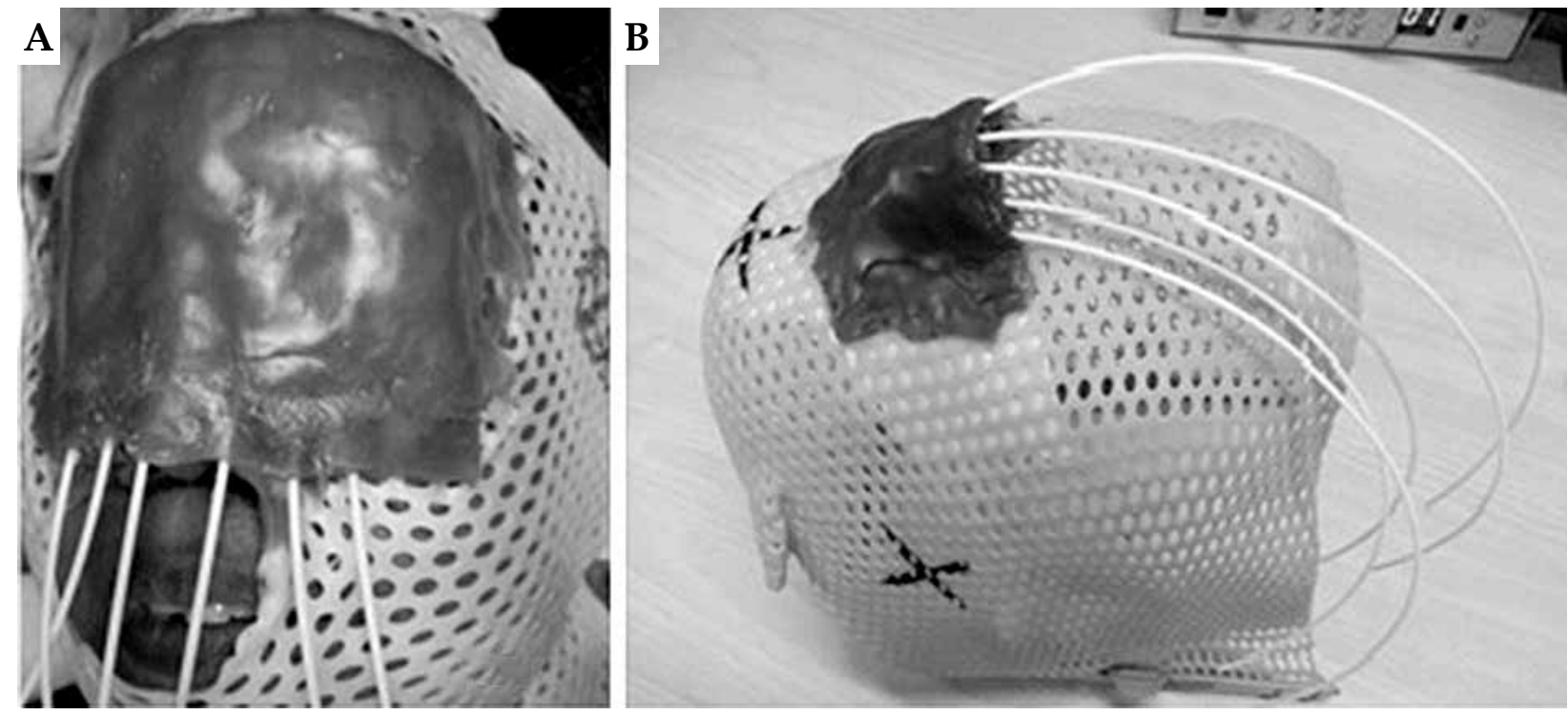

Fig. 2. Thermoplastic masks with customised moulds with plastic catheters in situ after preparation and before simulation on a patient (A) and without the patient (B)

The total dose prescribed was $42 \mathrm{~Gy}$ to the $100 \%$ isodose with an equivalent dose in $2 \mathrm{~Gy}\left(\mathrm{EQD}_{2}\right)$ of $47.25 \mathrm{~Gy}_{\alpha / \beta 10}$, delivered in twice daily fractions of 3.5 Gy each at least 6 hours apart for 12 fractions. The scheduled duration of treatment was 6 days. Treatment was delivered using iridium-192 ( $\left.{ }^{192} \mathrm{Ir}\right)$ with the GammaMed plus HDR afterloader unit (Varian Medical Systems).

The brachytherapy treatment plans of the selected patients were then analysed and various parameters (details given later) were recorded to evaluate the dose coverage, conformity and normal tissue sparing.

\section{Treatment planning for electron beam therapy}

The CT scans acquired for brachytherapy planning were transferred to the treatment planning system (Eclipse Version 15.5, Varian Medical Systems, Palo Alto, CA, USA) for electron beam planning. The primary brachytherapy bolus density was removed (changed to air equal density). For electron planning, the bolus was virtually added (Figure 3 ).

The PTVs for the brachytherapy plans were contoured similar to the PTV for the electron plans and no additional margins were given. The OARs were also contoured similar to those in the brachytherapy plans.

For each patient 2 or 3 electron beam plans (created using the Monte Carlo algorithm) using different energies $(6,9$ or $12 \mathrm{MeV}$ ) and different bolus thicknesses (5 or $10 \mathrm{~mm}$ water density bolus) were generated and the best plans, with respect to optimal coverage of the targets and acceptable doses to the OARs, were selected for analysis.

Parameters similar to those recorded for the brachytherapy plans were also recorded for the electron plans.

\section{Plan comparison}

The PTV coverage was assessed by recording the minimal doses to $90 \%, 95 \%$ and $5 \%$ of the PTV $\left(D_{90}, D_{95}, D_{5}\right)$, volumes of PTV receiving 90\%,100\%, 125\%, and $150 \%$ of prescribed dose $\left(\mathrm{VPTV}_{90}, \mathrm{VPTV}_{100}, \mathrm{VPTV}_{125}, \mathrm{VPTV}_{150}\right)$ defined as the percentage of the whole PTV volume, dose homogeneity index (DHI) and dose non-uniformity ratio (DNR) (only for BT plans). We calculated the conformal index for 90 and $100 \%$ of the prescribed dose $\left(\mathrm{COIN}_{90}\right.$, $\left.\mathrm{COIN}_{100}\right)$. For OARs, minimum doses to the most exposed $2 \mathrm{cc}$ and $0.1 \mathrm{cc}$ of the organs were noted. The OARs for which the doses were recorded were underlying bones, eyeballs, lens and overlying skin.

All doses were expressed as a percentage of the total dose. The prefix BT or Electron before the name of the parameter applies to the BT or Electron plan. The following definitions for parameters were used:

- $\mathrm{COIN}_{100}=\left(\mathrm{VPTV}_{100(\mathrm{cc})} / \mathrm{VPTV}_{(\mathrm{cc})}\right) \times\left(\mathrm{VPTV}_{100(\mathrm{cc})} /\right.$ $\left.\mathrm{V}_{100(\mathrm{cc})}\right)$ [16],

- $\mathrm{COIN}_{90}=\left(\mathrm{VPTV}_{90(\mathrm{cc})} / \mathrm{VPTV}_{(\mathrm{cc})}\right) \times\left(\mathrm{VPTV}_{90(\mathrm{cc})} / \mathrm{V}_{90(\mathrm{cc})}\right)$ [16],

- $\quad \mathrm{DNR}=\mathrm{V}_{150[\mathrm{cc}]} / \mathrm{V}_{100[\mathrm{cc}]}[17]$,

- BT-DHI inside PTV $=\left(\mathrm{VPTV}_{100[\mathrm{cc}]}-\mathrm{VPTV}_{150 \text { [cc]}}\right) /$ VPTV $_{100[\mathrm{ccl}}[17]$,

- Electron-DHI $=\mathrm{D}_{5} / \mathrm{D}_{95}$ [18],

where $\mathrm{V}_{90}, \mathrm{~V}_{100}$ and $\mathrm{V}_{150}$ are the volumes of PTV receiving $90 \%, 100 \%$ and $150 \%$ of the prescribed dose respectively.

\section{Data analysis}

The normal distribution of the data was analysed using the Shapiro-Wilk test. For comparison of the data, the $t$-test for paired samples or Wilcoxon's test was used depending on the normality of the data. All statistical analyses were performed using IBM SPSS v23. Results were considered significant if the $p$ value $<0.05$.

\section{Results}

The results are summarised in Tables 1 and 2 .

For BT plans, the average $\mathrm{D}_{0.1 \mathrm{cc}}$ for overlying skin was $171.8 \%$ (SD $19.7 \%$ ). We tried not to exceed $200 \%$ of the 

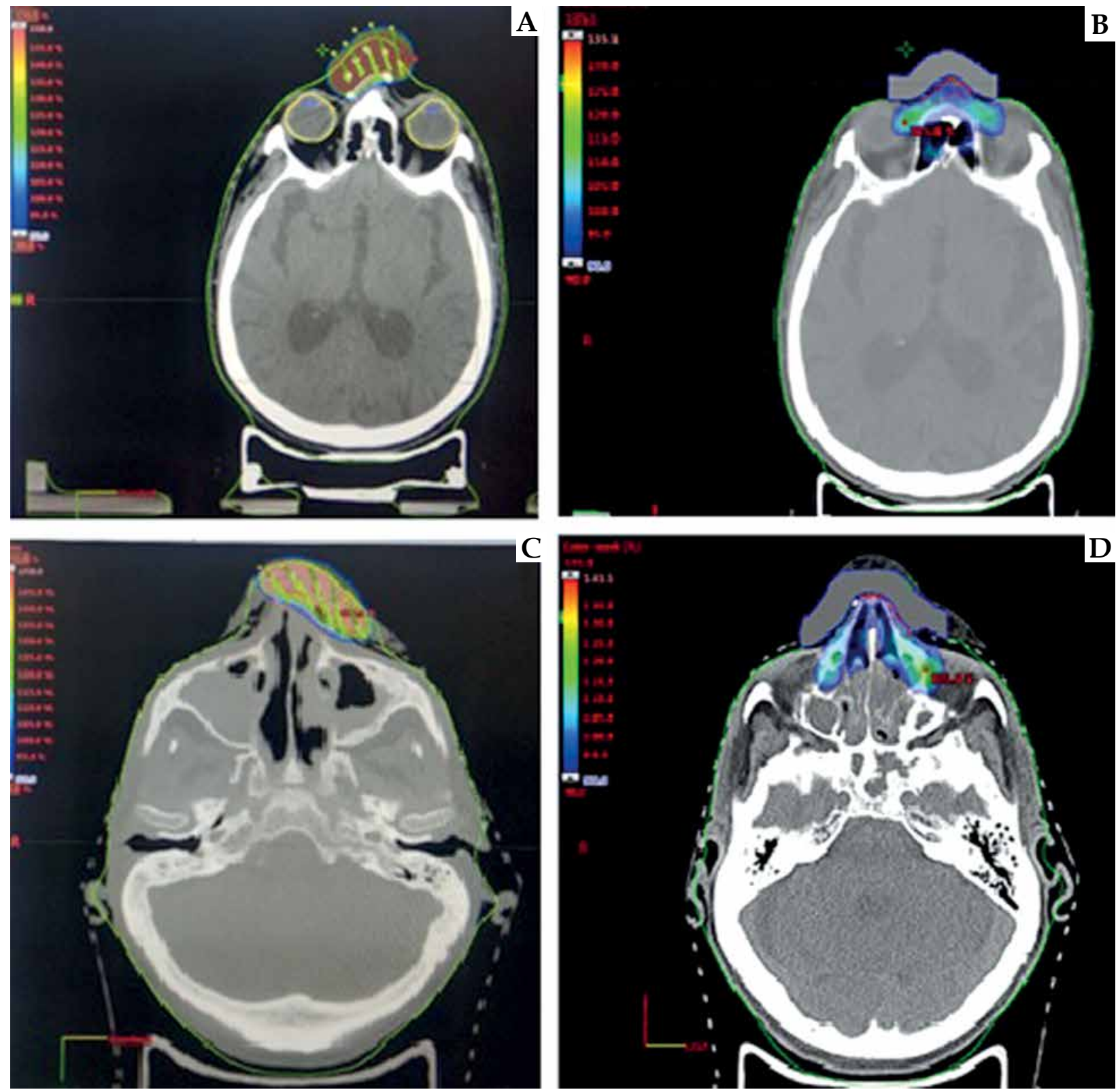

Fig. 3. Comparison of dose colour wash of customised surface mould brachytherapy plan and electron beam plan for a patient with NMSC over nasal bridge (A, B) and over the nasal skin (C, D)

prescribed dose to the most exposed $0.1 \mathrm{cc}$ of skin surface inside the PTV. This goal was achieved in all patients. Because of this very high dose to the skin, most of the patients developed significant acute and late skin toxicities (Figure 4). For Electron plans, in no patient did the dose to the most exposed $0.1 \mathrm{cc}$ of the overlying skin inside the PTV exceed $200 \%$ of the prescribed dose.

\section{Comparison of brachytherapy and electron plans}

We observed no significant differences in $\mathrm{D}_{90}$ and $\mathrm{D}_{95}$ between the BT and Electron plans, but there was a significantly higher mean $\mathrm{D}_{5}$ for the BT plans $(p<0.05)$. No significant differences were found in the mean $\mathrm{VPTV}_{90}$ and VPTV $_{100}$ but the mean VPTV $_{125}$ and VPTV $_{150}$ were significantly higher in the BT plans $(p<0.05$ for both). We found no difference in $\mathrm{COIN}_{100}$ but a significantly higher value was observed for $\mathrm{COIN}_{90}$ for the BT plans $(p<0.05)$.

For OARs, significantly higher doses were observed with Electron plans for most of the OARs except for $D_{0.1 c c}$ bones and $\mathrm{D}_{0.1 \mathrm{cc}}$ lens (no significant differences in these two). As for skin doses, the $\mathrm{D}_{0.1 \mathrm{cc}}$ was significantly higher for the BT plans $(p<0.05)$.

\section{Discussion}

In the literature, not many studies are present which deal with treatment of NMSCs of the nose and surrounding difficult areas with brachytherapy. In some of them, special applicators (e.g. Valencia, Leipzig, etc.) or electronic brachytherapy were used $[19,20,21]$. But they were not feasible for irregular surfaces. Experiences of using 
Table 1. Dosimetric comparison of the customized surface mould brachytherapy and electron beam plans

\begin{tabular}{|c|c|c|c|}
\hline Parameters & Brachytherapy & Electron & $P$-value \\
\hline Mean PTV vol. & \multicolumn{2}{|c|}{$2.31 \mathrm{cc} \pm 0.56$} & \\
\hline Mean $\mathrm{D}_{90}$ & $93.88 \% \pm 5.90 \%$ & $94.68 \% \pm 10.49 \%$ & 0.821 \\
\hline Mean $\mathrm{D}_{95}$ & $86.89 \% \pm 6.34 \%$ & $92.40 \% \pm 13.50 \%$ & 0.431 \\
\hline Mean $\mathrm{D}_{5}$ & $165.27 \% \pm 30.97 \%$ & $113.26 \% \pm 5.46 \%$ & 0.0003 \\
\hline Mean VPTV 90 & $92.90 \% \pm 6.48 \%$ & $94.37 \% \pm 8.28 \%$ & 0.398 \\
\hline Mean VPTV 100 & $81.773 \% \pm 11.77 \%$ & $68.56 \% \pm 31.24 \%$ & 0.158 \\
\hline Mean VPTV 125 & $41.95 \% \pm 23.38 \%$ & $0.344 \% \pm 0.59 \%$ & 0.012 \\
\hline Mean VPTV 150 & $15.825 \% \pm 20.12 \%$ & 0 & 0.023 \\
\hline Mean DHI & $0.81 \pm 0.23$ & $1.25 \pm 0.16$ & \\
\hline Mean DNR & $0.608 \pm 0.277$ & & \\
\hline $\mathrm{COIN}_{90}$ & $0.104 \pm 0.06$ & $0.057 \pm 0.033$ & 0.037 \\
\hline $\mathrm{COIN}_{100}$ & $0.1 \pm 0.08$ & $0.05 \pm 0.04$ & 0.075 \\
\hline
\end{tabular}

Table 2. Comparison of dose volume parameters of the organ at risk, between the surface mould and electron beam therapy plan

\begin{tabular}{lcccc}
\hline OARs & Parameters & Brachytherapy & Electron & $P$-value \\
\hline Bones & $\mathrm{D}_{0.1 \mathrm{cc}}$ & $81.87 \% \pm 14.62 \%$ & $119.06 \% \pm 7.02 \%$ & 0.528 \\
\cline { 2 - 4 } & $\mathrm{D}_{2 \mathrm{cc}}$ & $43.79 \% \pm 11.29 \%$ & $101.98 \% \pm 4.58 \%$ & 0.001 \\
\hline Eyeballs & $\mathrm{D}_{0.1 \mathrm{cc}}$ & $49.05 \% \pm 15.09 \%$ & $113.33 \% \pm 15.4 \%$ & 0.012 \\
\hline & $\mathrm{D}_{2 \mathrm{cc}}$ & $21 \% \pm 10.5 \%$ & $79.1 \% \pm 15.55 \%$ & 0.003 \\
\hline Lens & $\mathrm{D}_{0.1 \mathrm{cc}}$ & $31.2 \% \pm 13.1 \%$ & $62.99 \% \pm 35.06 \%$ & 0.163 \\
\hline Skin & $\mathrm{D}_{0.1 \mathrm{cc}}$ & $171.8 \% \pm 19.68 \%$ & $113.30 \% \pm 7.47 \%$ & 0.0003 \\
& $\mathrm{~V}_{200}$ & 0 patients & 0 patients &
\end{tabular}

custom mould brachytherapy are also scarce. A few studies have used them for treatment of skin cancers and the results have been promising, compared to other modes of radiation delivery $[13,15,22,23,24]$. In a few studies, 3D planning based custom mould brachytherapy was used and they showed very good dose distribution with respect to PTV coverage and OAR sparing. In one of the studies [25], surface mould based HDR brachytherapy showed very good results for cutaneous facial BCC and SCC but also showed high failure rates in tumours located over the nose. This emphasizes the inherent difficulty in treating lesions over small irregular surfaces.

Now coming to electrons as a modality of treatment of superficial skin cancers, especially on small irregular surfaces, the difficulty is that there is huge variation in the PDD and output parameters and they depend on the field size and the electron energy used [26]. Studies comparing electrons with surface mould brachytherapy for treatment of NMSC are rare. The studies $[27,28]$ which compared these two modalities directly concluded that local control, acute and late toxicities were comparable but brachytherapy had the advantage of better confor-

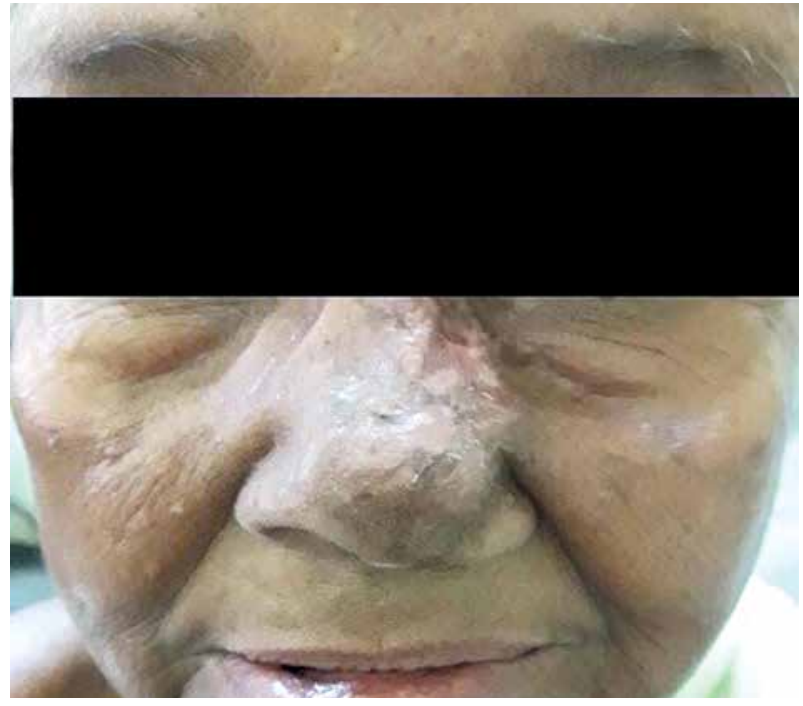

Fig. 4. Post-treatment skin hypopigmentation. 12 weeks after being treated with surface mould brachytherapy with hypopigmented area corresponding to skin surface receiving $>150 \%$ of the prescribed dose 
mity, shorter treatment times and cost effectiveness. However, in another study [29], brachytherapy had better cosmesis compared to EBRT. One study [17], which compared surface mould brachytherapy with EBRT, concluded that computed tomography-based surface mould brachytherapy for superficial lesions on irregular surfaces is superior to 3D-CRT EBRT in those locations, in terms of conformity and normal tissue sparing ability in medium to high doses.

In our study, we found very good PTV coverage with both electron and brachytherapy plan (as demonstrated by the non-significant differences in $\mathrm{D}_{90}, \mathrm{D}_{95}, \mathrm{VPTV}_{90}$ and VPTV $_{100}$ between the two modalities). The overdoses inside the PTV for BT plans were acceptable $\left(\mathrm{VPTV}_{125} 42 \%\right.$, $\mathrm{VPTV}_{150} 15.8 \%$ ) but they were still significantly higher than the electron plans. This translates into higher heterogeneity inside the PTV with BT.

This study showed that conformity was much better with brachytherapy (significantly different $\mathrm{COIN}_{90}$ values). This finding corroborates other studies $[17,27]$. However, the $\mathrm{COIN}_{100}$ values were not significantly different.

The homogeneity indices were calculated using different formulas for the two modalities. Therefore direct comparison of this parameter is not ideal and was not done. However, considering this parameter and taking into account the greater overdosages inside the PTV for the BT plans, we can say that there was significant heterogeneity in the BT plans.

OAR sparing was much better with the brachytherapy plans, although we did not use any lead shielding for the eyes in the Electron plans. This might have been a potential cause of much higher OAR doses in the electron plans. One very significant finding was the very high doses to the overlying skin inside the PTV with the brachytherapy plans (significantly higher $\mathrm{D}_{0.1 c c}$ doses). This can lead to severe acute reactions and even late skin toxicity. But considering the fact that these are superficial skin tumours, whether this finding is good or bad remains debatable.

Dose calculations were done using TG-43 formalism for the brachytherapy plans and the Monte Carlo algorithm for the electron plans. Both of them have some limitation with respect to small field dosimetry $[26,30]$. So errors in dose calculation could have affected the results.

This study was a dosimetric study only and the results need to be validated in prospective trials directly comparing these two modalities. Moreover, the sample size for this study is very small and this makes it difficult to draw definitive conclusions based on the results.

\section{Conclusions}

Surface-mould HDR BT might offer a better conformity index and coverage than electron EBRT for skin non-melanoma cancers of the nose, with better sparing of the lens, eyeballs, bone and cartilage at the price of higher maximum skin dose, which can be relevant for skin toxicity, even for adjuvant doses. Prospective clinical studies are needed to validate our small sampled dosimetry results. However, data comparing these two modalities are limited and this study aims to provide a small insight into this topic.

\section{Disclosure}

The authors report no conflict of interest.

\section{References}

1. Bray F, Ferlay J, Soerjomataram I et al. Global cancer statistics 2018: GLOBOCAN estimates of incidence and mortality worldwide for 36 cancers in 185 countries. CA Cancer J Clin 2018; 68: 394-424.

2. Gloster HM, Neal K. Skin cancer in skin of color. J Am Acad Dermatol 2006; 55: 741-760; quiz 761-764.

3. Adinarayan M, Krishnamurthy SP. Clinicopathological evaluation of nonmelanoma skin cancer. Indian J Dermatol 2011; 56: 670-672.

4. Bath-Hextall F, Bong J, Perkins W et al. Interventions for basal cell carcinoma of the skin: systematic review. BMJ 2004; 329: 705.

5. Mendenhall WM, Mancuso AA, Kirwan JM et al. Skin. In: Perez and Brady's principles and practice of radiation oncology. Wolters Kluwer Health, New York 2018.

6. Ouhib Z, Kasper M, Perez Calatayud J et al. Aspects of dosimetry and clinical practice of skin brachytherapy: The American Brachytherapy Society working group report. Brachytherapy 2015; 14: 840-858.

7. Pierquin BF. Wilson J, Chassagne D. Modern brachytherapy. Am J Clin Oncol 1988; 11: 685.

8. Mazeron JJ, Chassagne D, Crook J et al. Radiation therapy of carcinomas of the skin of nose and nasal vestibule: a report of 1676 cases by the Groupe Europeen de Curiethérapie. Radiother Oncol 1988; 13: 165-173.

9. Crook JM, Mazeron JJ, Marinello G et al. Interstitial iridium 192 for cutaneous carcinoma of the external nose. Int J Radiat Oncol Biol Phys 1990; 18: 243-248.

10. Linos E, VanBeek M, Resneck JS. A sudden and concerning increase in the use of electronic brachytherapy for skin cancer. JAMA Dermatol 2015; 151: 699-700.

11. Ashby MA, Pacella JA, de Groot R et al. Use of a radon mould technique for skin cancer: results from the Peter MacCallum Cancer Institute (1975-1984). Br J Radiol 1989; 62: 608-612.

12. Gerbaulet A, Pötter R, Mazeron J et al. The GEC ESTRO Handbook of Brachytherapy. ESTRO Publisher, Brussels 2002.

13. Guix B, Finestres F, Tello J et al. Treatment of skin carcinomas of the face by high-dose-rate brachytherapy and custom-made surface molds. Int J Radiat Oncol Biol Phys 2000; 47: 95-102.

14. Skowronek J. Brachytherapy in the treatment of skin cancer: an overview. Postepy Dermatol Alergol 2015; 32: 362-367.

15. Frakulli R, Galuppi A, Cammelli $S$ et al. Brachytherapy in non melanoma skin cancer of eyelid: a systematic review. J Contemp Brachytherapy 2015; 6: 497-502.

16. Feuvret L, Noël G, Mazeron JJ et al. Conformity index: A review. Int J Radiat Oncol 2006; 64: 333-342.

17. Kuncman $\succeq$, Kozłowski S, Pietraszek A et al. Highly conformal CT based surface mould brachytherapy for non-melanoma skin cancers of earlobe and nose. J Contemp Brachytherapy 2016; 3: 195-200.

18. Kataria $T$, Sharma K, Subramani V et al. Homogeneity Index: An objective tool for assessment of conformal radiation treatments. J Med Phys 2012; 37: 207-213.

19. Gauden R, Pracy M, Avery AM et al. HDR Brachytherapy for Superficial Non-Melanoma Skin Cancers: HDR brachytherapy for superficial NMSC. J Med Imaging Radiat Oncol 2013; 57: 212-217. 
20. Pons-Llanas O, Ballester-Sánchez R, Celada-Álvarez FJ et al. Clinical implementation of a new electronic brachytherapy system for skin brachytherapy. J Contemp Brachytherapy 2015; 6: 417-423.

21. Tormo A, Celada F, Rodriguez $S$ et al. Non-melanoma skin cancer treated with HDR Valencia applicator: clinical outcomes. J Contemp Brachytherapy 2014; 6: 167-172.

22. Olek D, El-Ghamry MN, Deb N et al. Custom mould applicator high-dose-rate brachytherapy for nonmelanoma skin cancer - an analysis of 273 lesions. Brachytherapy 2018; 17: 601-608.

23. Mukherji A, Mourougan S, Saravannan K et al. Dosimetric analysis and clinical outcomes in CT-based mould brachytherapy in early oral cancers in patients unfit for surgery. J Contemp Brachytherapy 2015; 7: 147-153.

24. Budrukkar A, Dasgupta A, Pandit P et al. Clinical outcomes with high-dose-rate surface mould brachytherapy for intra-oral and skin malignancies involving head and neck region. J Contemp Brachytherapy 2017; 3: 242-250.

25. Maroñas M, Guinot JL, Arribas L et al. Treatment of facial cutaneous carcinoma with high-dose rate contact brachytherapy with customized moulds. Brachytherapy 2011; 10: 221-227.

26. Deiab N, Kader S, Tolba A et al. Dosimetry for small, irregular and rectangular field size for electron beam therapy. Med J Cairo Univ 2015; 83: 733-738.

27. Vyas S, Palaniswaamy G, Massingill B et al. Electrons versus surface brachytherapy for non-melanoma skin cancer a matched pair analysis. Int J Radiat Oncol 2014; 90: S118.

28. Haseltine JM, Parker M, Wernicke AG et al. Clinical comparison of brachytherapy versus hypofractionated external beam radiation versus standard fractionation external beam radiation for non-melanomatous skin cancers. J Contemp Brachytherapy 2016; 3: 189-194.

29. Zaorsky NG, Lee CT, Zhang E et al. Skin CanceR Brachytherapy vs External beam radiation therapy (SCRiBE) meta-analysis. Radiother Oncol 2018; 126: 386-393.

30. Granero D, Perez-Calatayud J, Vijande J et al. Limitations of the TG-43 formalism for skin high-dose-rate brachytherapy dose calculations: Limitations of skin HDR brachytherapy dose calculations. Med Phys 2014; 41: 021703. 